\title{
Stress-Strain and Creep Characteristics of HAF Black- Loaded NBR/EPDM Rubber Blends
}

\author{
M. H. Abd-EI Salam
}

Phys. Depart., Faculty of Education, Ain Shams University, Cairo,
Egypt.

The effect of carbon black concentration on the stress-strain and creep characteristics of NBR/EPDM blend was studied. The mechanical parameters obtained from the stress-strain and the creep measurements may suggest the formation of a compact structure around the concentration of $40 \mathrm{phr} H A F$ black. The stress- strain behaviour of the composites under test is fairly consistent with the standard linear solid model. All the obtained data from the creep measurements are discussed within the frame of Burgers model.

\section{Introduction:}

The determination of deformation mechanisms and the modeling of the visco-elastic behaviour of polymeric materials have recently received a considerable interest due to the extensive use of polymers in a broad range of applications, including the electronic systems, aerospace and automotive industries and consumer appliances. The mechanical properties of the heterogeneous polymeric systems including polymer blends depend on the microstructure of the sample, the size and shape of the domains, and the interactions among the components [1,2]. The reinforcement of a polymer by finely dispersed fillers was discussed previously [3] from the view point of its relationship to the degree of molecular orientation at break which is dependent on the strain developed at the break point and the nature of the stressed state. It was also found that, for this relationship, the effect of filler on the strength properties can vary under different conditions of fracture.

Banerjee and Millns[4] performed a systematic study on the physical and mechanical properties of crosslinked rubber blends. They found that, blending of crosslinked Butyl rubber(IIR) elastomers with (NR,SBR,EPDM) rubber, significantly increased their aging performance and ozone resistance, as well as deterioration of some physical properties such as tensile strength, tear strength, elongation, compression set, minimum viscosity, maximum rheometric torque and die swell. They also confirmed that the magnitude of these effects varied according to the composition of the rubber blends. 
Abd-El Salam et al. [5] studied the effect of the vulcanizing system on the static and dynamic mechanical properties of butyl rubber/ethylene propylene diene monomer-general purpose furnace black-(GPF) blends. They applied the classical theory of elasticity to show the mechanical behavior of the rubberpolymer blend and to calculate the degree of crosslinking. From the dynamic mechanical measurements, the elastic modulus, internal friction, and thermal diffusivity were calculated. The observed variations were explained in view of the role played by both the vulcanizing system and the reinforcing carbon black.

Abu-Abdeen [6] studied the degradation of the mechanical properties of the composite of Styrene Butadiene Rubber (SBR) blended with natural rubber (NR) loaded with $40 \mathrm{phr}$ of high abrasion furnace (HAF) carbon black and different concentrations of paraffin wax. From the stress-strain curves, Young's modulus was found to decrease with increasing the amount of added paraffin wax. The average molecular weight between crosslinks and the number of effective plastic chains per unit volume were calculated.

Creep relaxation in carbon black loaded NBR-Neoprene composite was studied by Fahim et al. [7]. A four-element model has been tested to account for the creep relaxation in a technological material composed of a common rubber and different ingredients. Interesting results have been obtained and discussed within the frame of the above mentioned model.

The stress-strain variations $[8,9]$ were used to investigate the effect of low-structure (HAF) on the mechanical properties of rubber blend of styrene butadiene rubber and natural rubber $(\mathrm{SBR}+\mathrm{NR})$ of equal parts.

The aim of this work is to carry out a systematic study for the mechanical properties of NBR/EPDM blends loaded with carbon black. The effect of carbon black on the mechanical properties is considered. The consistence of the stress- strain behaviour with the mechanical models which describe the visco-elastic behaviour is tried. The creep measurements are investigated in view of Burgers model.

\section{Experimental:}

\subsection{Materials}

The composition of all samples in this work is expressed in parts per hundred part of rubber by weight (phr). The blend formulation contains NBR (50 phr), EPDM (50 phr), ZnO (5 phr), stearic acid (2 phr), processing oil (10 phr), N-cyclohexyl-2-benzthiozyle sulfenamide (1.5 phr), and different contents of HAF black up to $50 \mathrm{phr}$. The vulcanizing system used for these samples contains: elemental sulfur (2.5 phr); tetramethylthiuram disulfide TMTD (1 
phr) and Dibenzthiazyl disulphide MBTS (1.5 phr). The composites were prepared on a two-roll mill $170 \mathrm{~mm}$ in diameter with working distance $300 \mathrm{~mm}$, speed of slow roll $24 \mathrm{rev}$./ min and gear ratio 1.25. The rubber samples were left for at least 48 hours before being vulcanized. The vulcanization process was conducted at $160 \pm 2{ }^{\circ} \mathrm{C}$ under a pressure of $4 \mathrm{MPa}$ for $20 \mathrm{~min}$.

\subsection{Measurements}

Dumbell shaped samples were cut from the vulcanized sheets by a fine edge steel die with a constant width of $4 \mathrm{~mm}$. The thickness of the test sample was determined with a dial gauge. The apparatus used for stress-strain and creep measurements was described previously elsewhere [10]. The samples were stretched at a constant speed $(10 \mathrm{~mm} / \mathrm{min})$ adjusted by means of dc motor. The applied force was recorded continuously during the extension of the sample up to rupture. The elongation in the creep test was measured with a dial gauge of sensitivity $0.01 \mathrm{~mm}$.

\section{Results and Discussion:}

\subsection{Stress-strain}

The nominal stress-strain curves of HAF-loaded NBR/EPDM (50: 50) rubber blend were measured and the experimental results (points) obtained are shown in Fig. (1).This figure shows the conventional behaviour of thermosetting materials. Besides, the solid lines show the theoretical data obtained by applying the standard linear solid model represented by Eqn. (2).

From Fig. (1), it is clear that, the stiffness as governed by the slope of the initial linear part increases with increasing the concentration of carbon black which acts as reinforcing filler. Accordingly, the elongation at break decreases.

The mechanical stress-strain parameters; modulus of elasticity $E$, rupture strain $\varepsilon_{R}$ and rupture stress $\sigma_{R}$ of the samples as deduced from the stress-strain curves of Fig. (1) were found to be appreciably affected by increasing carbon black concentration. Figure (2) shows the carbon black concentration dependence of these parameters. In general, it is clear that $\mathrm{E}$ increases with increasing carbon black content and reaches a maximum value of $2.21 \mathrm{MPa}$ around $40 \mathrm{phr}$ of HAF black. On the other hand, $\varepsilon_{R}$ shows a minimum around the same concentration. The initial decrease in $\varepsilon_{R}$ and the observed increase of rupture stress $\sigma_{R}$ is probably due to entanglement of macromolecular chains by the introduction of some physical crosslinking between them and carbon black particles. Also, the subsequent formation of compact structure [11] with increasing the concentration of HAF black might be responsible for the minimum in $\varepsilon_{R}$ and the maximum of $\sigma_{R}$ observed around $40 \mathrm{phr}$ of HAF black. Such compact structure 
would become looser by adding excess carbon black leading to the maximum $\varepsilon_{R}$ and minimum $\sigma_{R}$ above $40 \mathrm{phr}$ carbon black content.

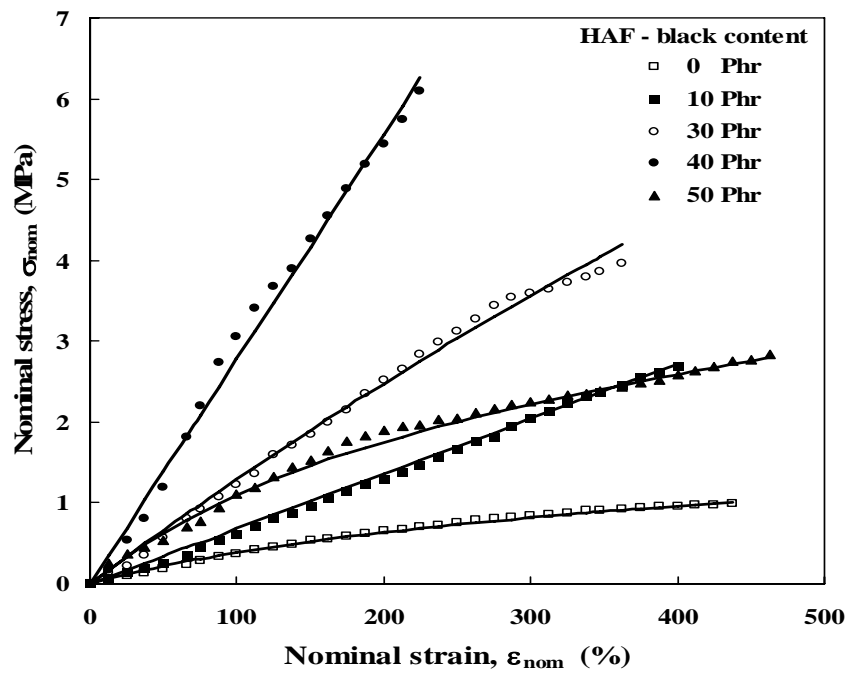

Fig.(1): Stress-strain curves for (NBR/EPDM) blends loaded with different concentrations of HAF black, the points represent the experimental data and the solid lines represent the standard linear solid model (Eqn. (2)).

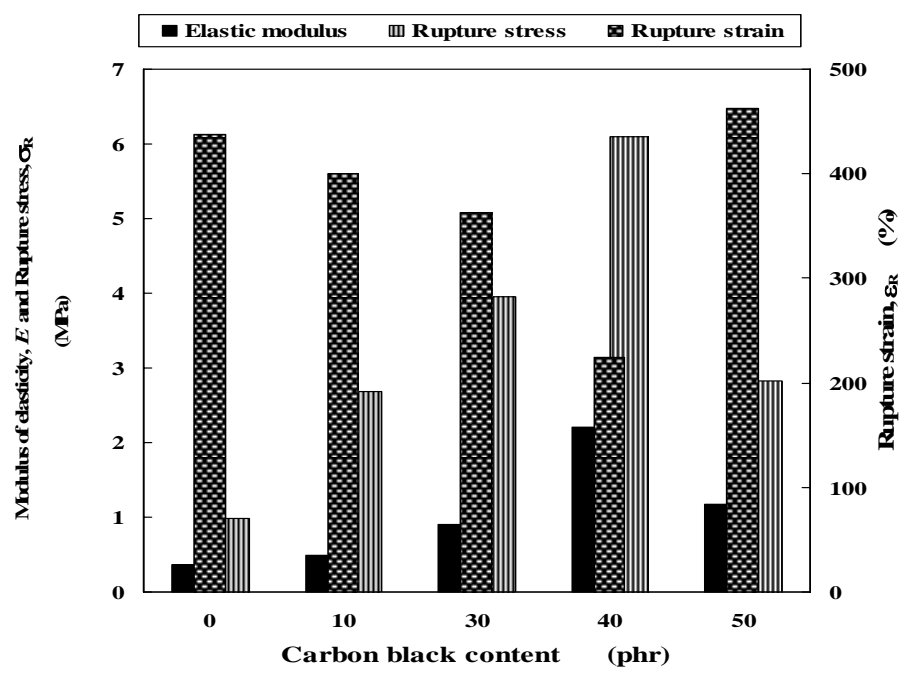

Fig. (2): Dependence of $(E),\left(\varepsilon_{R}\right)$ and $\left(\sigma_{R}\right)$ on the carbon black concentration for (NBR/EPDM) blends.

The stress-strain curve of polymers is often mathematically described by models indicating the visco-elastic behaviour of these polymers $[12,13]$. In the standard linear solid model, the visco-elastic behavior of polymers is described by a spring (with elastic modulus E) and a dashpot (with damping 
constant or viscosity $\boldsymbol{\eta}$ ) in series with each other, both of which are in parallel with a long spring (with elastic modulus E') as shown in Fig. (3). For this model the governing constitutive relation is:

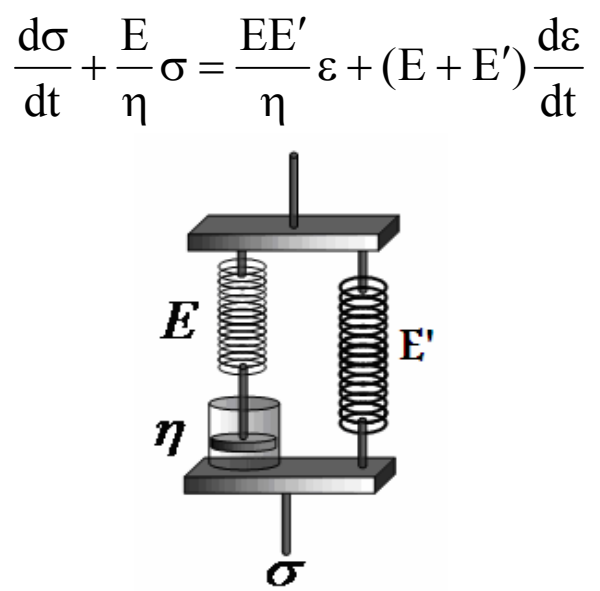

Fig. (3). The standard linear solid model used to simulate the stress strain behaviour of $\mathrm{HAF} /(\mathrm{NBR} / \mathrm{EPDM} /)$ composites.

To describe stress-strain curves in tensile testing with a constant strain rate, $(\gamma=d \varepsilon / d t)$ by using this model, the solution of equation (1) can be written as:

$$
\sigma=\eta \gamma\left[1-\mathrm{e}^{-\left(\frac{\mathrm{E} \varepsilon}{\eta \gamma}\right)}\right]+\mathrm{E}^{\prime} \varepsilon
$$

This equation contains three fitting parameters, $E, E^{\prime}$ and $\eta$. The application of this model is shown by the solid lines in Fig. (1) and the fitting parameters in Eqn. (2) are listed in table (1). There is an agreement between the values obtained from the theoretical model and the experimental data of the stress-strain curves. The fitting parameters $E, \eta$ and $E^{\prime}$ increase with increasing carbon black concentration from 0 to $40 \mathrm{phr}$. In the sample containing $50 \mathrm{phr}$ HAF-black, the values of the parameters decrease in consistance with the suggested formation of a compact structure. From table (1), it is also clear that the calculated values of the spring elastic modulus $E$ of the theoretical model are very close to those obtained experimentally from the slopes of the initial linear portions of the stress - strain curves. This indicates that the series spring in this model $E$ plays the dominant role in the early stage of the stress - strain curves, i.e. before reaching the yield point. While, the 
parallel spring and thus the modulus $E^{\prime}$ plays the dominant role after the yield point of the stress - strain curve.

The significance of the fitting parameter $E^{\prime}$ indicates that there is a significant increase in stress as a function of strain beyond the yield point. During post yield extension, the chain segments tend to become oriented along the stress axis and as a result, a structure may be obtained which approaches that of a semi-crystalline material. This is the so called "strain-induced crystallization" [15]. From table (1), it is also noticed that $E^{\prime} \cong E / 3$, therefore, the fitting parameter $E$ represents the Young's modulus while $E^{\prime}$ can be considered as the shear or rubbery modulus [16]. Besides, the values of $\eta$ as calculated from this fitting are comparable with those of the viscosity $\eta_{2}$ as calculated from the forward creep curves which will be discussed later [see table (2)].

Table (1): The fitting parameters calculated from the standard linear solid model for the investigated composites and the experimental values of the elastic moduli.

\begin{tabular}{ccccc}
\hline $\begin{array}{c}\text { Carbon black } \\
\text { content (phr) }\end{array}$ & $\begin{array}{c}E \\
(\mathrm{MPa})\end{array}$ & $\begin{array}{c}\eta \\
(\mathrm{GPa} . \mathrm{sec})\end{array}$ & $\begin{array}{c}E^{\prime} \\
(\mathrm{MPa})\end{array}$ & $\begin{array}{c}E_{\text {exp }} \\
(\mathrm{MPa})\end{array}$ \\
\hline 0 & 0.39 & 3.30 & 0.11 & 0.37 \\
10 & 0.60 & 16.30 & 0.23 & 0.49 \\
30 & 1.13 & 24.70 & 0.40 & 0.91 \\
40 & 2.24 & 56.76 & 0.88 & 2.21 \\
50 & 1.16 & 12.05 & 0.25 & 1.17 \\
\hline
\end{tabular}

\subsection{Creep and Creep Recovery}

Burgers model, which is a combination of Maxwell and Kelvin-Voigt elements [17], is one of the most used models to predict the creep behaviour because it is described by a differential equation whose response to an applied stress can be easily solved analytically [18]. The total elongation of the model which is equivalent to that of the test specimen may be considered as the sum of three components [15]; i.e.

$$
\varepsilon=\frac{\sigma_{\circ}}{E_{1}}+\frac{\sigma_{\circ}}{E_{2}}\left(1-\exp \frac{-t}{\tau}\right)+\frac{\sigma_{\circ}}{\eta_{3}} t
$$

where the first term gives the initial elongation, the second term and the third 
one give the later elongation from the spring of modulus $E_{2}$ and the dashpot of viscosity $\eta_{2}$ in parallel ; moreover, it comes from the dashpot with viscosity $\eta_{3}$ in series, $\sigma_{0}$ is the applied stress, and the retardation time is given by

$$
\tau=\frac{\eta_{2}}{\mathrm{E}_{2}} \cdot
$$

When the stress is suddenly removed at certian time $\left(t_{1}\right)$, the reduction in elongation is equal to what is called the instantaneous strain $\sigma_{0} / E_{1}$, while the subsequent recoverable creep is given by:

$$
\varepsilon=\varepsilon_{2} \exp \frac{-\left(\mathrm{t}-\mathrm{t}_{1}\right)}{\tau}+\frac{\sigma_{\circ}}{\eta_{3}} \mathrm{t}_{1}
$$

where $\varepsilon_{2}$ is given by :

$$
\varepsilon_{2}=\frac{\sigma_{\circ}}{E_{2}}\left(1-\exp \frac{t_{1}}{\tau}\right)
$$

Therefore, the following sections aim to study the factors that affect the creep mechanisms, such as the applied axial stress considered here as ranging from 0.98 to $3.92 \mathrm{MPa}$ and carbon black concentration up to $50 \mathrm{phr}$. Observations were recorded during forward creep and backward creep, for recoverable strain, memory function and the retardation time. Figure (4) represents the forward and backward creep of some selected curves under different values of the applied stress (at room temperature $30^{\circ} \mathrm{C}$ ) for samples containing $30 \mathrm{phr}$ of carbon black as representative example. Fig. (5) represents creep and creep recovery curves for samples containing 30,40 and $50 \mathrm{phr}$ of carbon black under the applied stress $(\sigma=1.96 \mathrm{MPa})$.

\section{(i) Forward Creep}

It is observed from Fig.(5) that, the increase of HAF black content largely affects the total resulting strain and some parameters, such as instantaneous strain $\left(\varepsilon_{0}=\sigma / E_{1}\right)$ and the strain rate $\varepsilon_{\text {steady }}$. The applied stress shows an important effect reflected on the set of curves of fig.4; where it raises the instantaneous strain and the strain rate. After 120 minutes, at which the steady state is reached, the applied stress is removed and the subsequent strain is recorded with time. There was always a sudden drop in strain followed by exponential decrease [19] attributed eventually to the visco-elastic deformation irrecoverable strain i.e. $\left(\sigma_{0} / \eta_{3}\right) t_{1}$.

The increase in the creep strain by increasing the applied stress, Fig.(4) was attributed to the stress enhancement of the translational motion and the increased mobility of the flexible and rigid molecular chain segments. This enhancement has been achieved by the movement and rearrangement of carbon black aggregates in the direction of creep strain. Table (2) represents the 
variation of the conventional elastic modulus $\mathrm{E}_{1}=\sigma / \varepsilon_{0}$, high elasticity modulus $\mathrm{E}_{2}=\sigma / \varepsilon_{\mathrm{el}}$, the viscosity,

$$
\eta_{2}=\frac{\sigma}{(\mathrm{d} \varepsilon / \mathrm{dt})},
$$

for steady creep and the activation volume q [12] with HAF black content under different applied stresses.

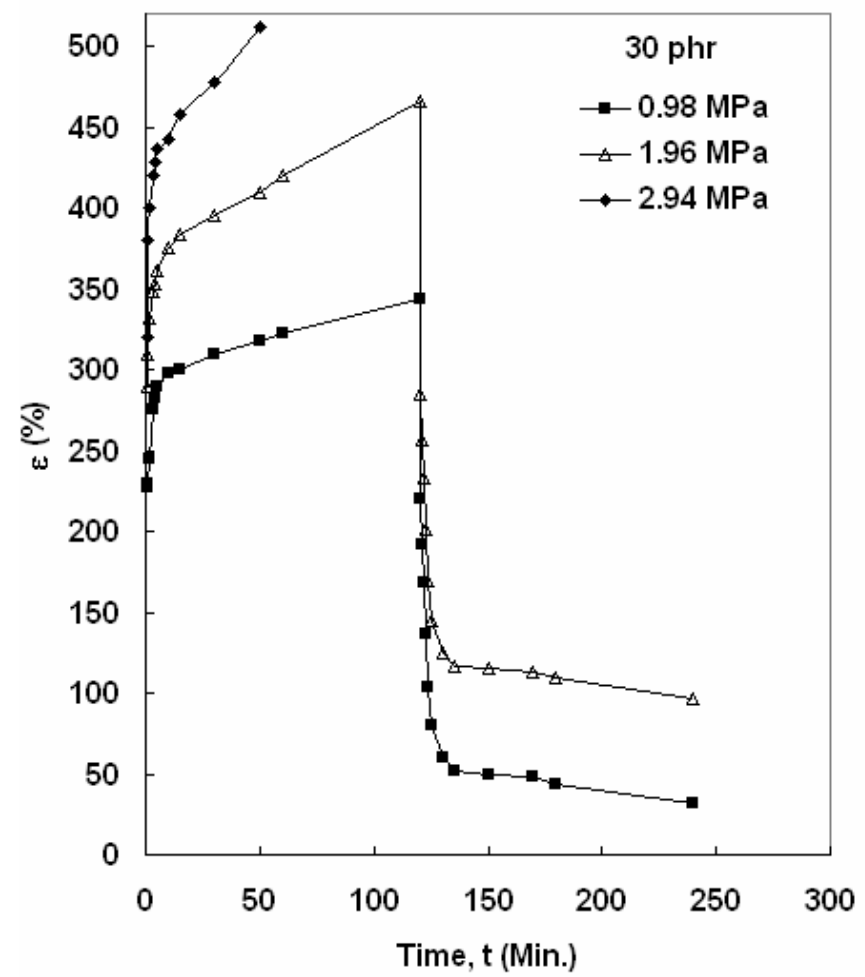

Fig. (4): Creep and creep recovery curves for samples containing $30 \mathrm{phr}$ of carbon black under different applied stresses. 


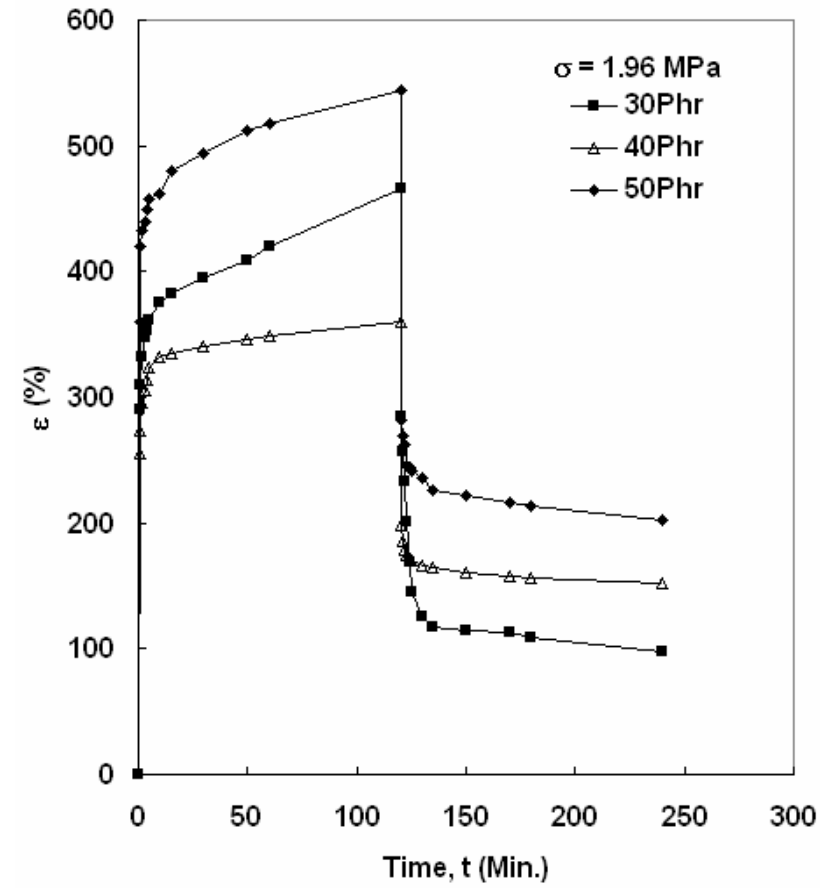

Fig. (5): Creep and creep recovery curves for samples containing 30, 40 and $50 \mathrm{phr}$ of carbon black under the applied stress $(\sigma=1.96 \mathrm{MPa})$.

Table (2): The dependence of $E_{1}, E_{2}, \eta_{2}$ and $q$ on HAF content under different applied stresses.

\begin{tabular}{|c|c|c|c|c|c|}
\hline $\begin{array}{l}\text { Carbon black } \\
\text { Content (phr) }\end{array}$ & $\begin{array}{c}\sigma \\
(\mathrm{MPa})\end{array}$ & $\begin{array}{c}\mathrm{E}_{1} \\
(\mathrm{MPa})\end{array}$ & $\begin{array}{c}\mathrm{E}_{2} \\
(\mathrm{MPa})\end{array}$ & $\begin{array}{c}\eta_{2} \\
(\mathrm{GPa} \cdot \mathrm{sec})\end{array}$ & $\underset{\left(m^{3}\right)}{q}$ \\
\hline \multirow{3}{*}{30} & 0.98 & 0.43 & 1.56 & 29.43 & \multirow{3}{*}{$1.24 \times 10^{-27}$} \\
\hline & 1.96 & 0.67 & 2.45 & 28.82 & \\
\hline & 2.94 & 0.92 & 2.60 & 26.49 & \\
\hline \multirow{3}{*}{40} & 1.96 & 0.77 & 2.68 & 62.16 & \multirow{3}{*}{$6.21 \times 10^{-28}$} \\
\hline & 2.94 & 1.06 & 3.72 & 60.15 & \\
\hline & 3.92 & 1.31 & 4.08 & 61.33 & \\
\hline \multirow{3}{*}{50} & 0.98 & 0.50 & 1.48 & 17.19 & \multirow{3}{*}{$1.11 \times 10^{-27}$} \\
\hline & 1.96 & 0.54 & 1.81 & 14.96 & \\
\hline & 2.94 & 0.57 & 1.84 & 11.44 & \\
\hline
\end{tabular}

From Table (2), it is clear that $E_{1}, E_{2}$ and $\eta_{2}$ increase with increasing carbon black concentration under constant applied stress showing maxima at carbon black content $40 \mathrm{phr}$. This insures the suggestion mentioned before that a compact structure may be formed at this carbon content. 
Figure (6) shows the stress dependence of strain rate $\varepsilon_{\text {steady }}$, from which the activation volume $q$ was calculated according to the following equation [20], $\varepsilon_{\text {st. }}=A \exp [-(\mathrm{U}-\mathrm{q} \sigma) / \mathrm{kT}]$. The calculated values of $\mathrm{q}$, in table (2), show that the addition of the fillers lowers the activation volume $q$, into which the macromolecular chain may be translated or slipped through this volume during the creep process. This is attributed to the formation of chemical and physical cross-links with increasing HAF content, which restrict the motion of macromolecules, i.e. the formation of carbon black aggregates between the flexible chains segments act as obstacles to their sliding. So, the activation volume decreases with increasing HAF contents.

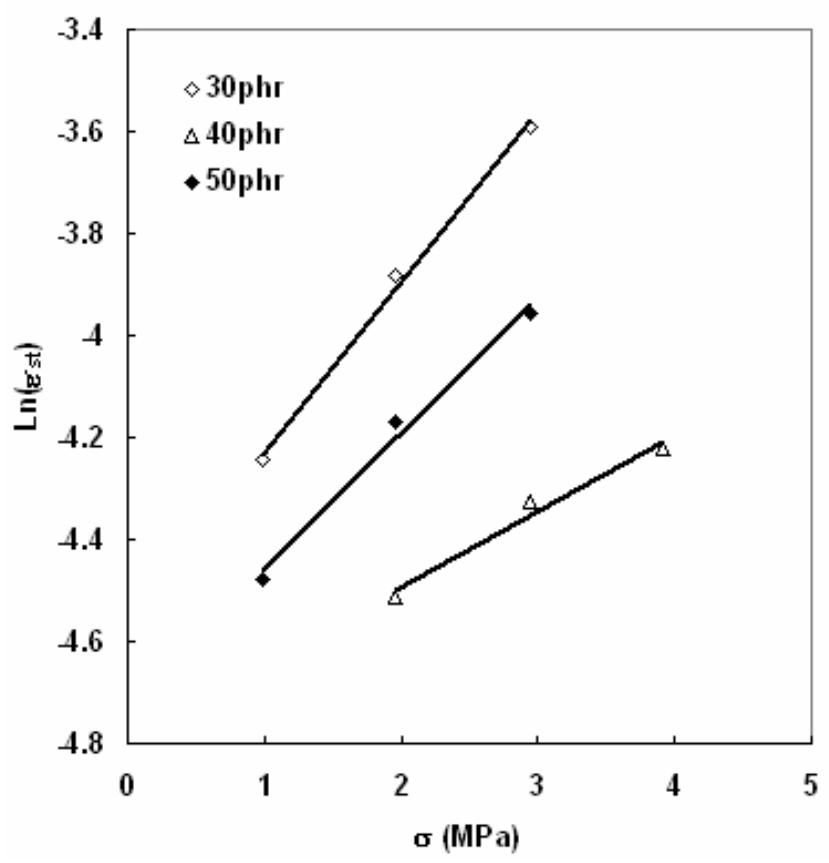

Fig. (6): Stress dependence of strain rate, $\mathcal{E}_{\text {steady }}^{\cdot}$

\section{(ii) Backward Creep}

The backward creep ,i.e., creep after removal of the applied stress at a time $t_{1}$ in the steady state region, is also useful in deducing several important parameters and studying their dependence on carbon black concentration, applied stress as well as vulcanization process [21,22].

Table (3) represents the variations of the retardation time for recoverable creep $\tau$ which was calculated from $\varepsilon_{\text {el.r }}=\mathrm{A} \exp (-\mathrm{t} / \tau)$ [20], memory function 
for recoverable deformation $\beta(\mathrm{t})$, relaxation strength $\Delta=\varepsilon_{\text {el.r. }} / \varepsilon_{\mathrm{r}}[13]$ and the viscosity $\eta_{3}$ at plastic deformation (irrecoverable strain) with HAF black content under different applied stresses.

Table (3): The dependence of $\tau, \beta(t), \Delta$ and $\eta_{3}$ on HAF concentration under different applied stresses.

\begin{tabular}{|c|c|c|c|c|c|}
\hline $\begin{array}{c}\text { Carbon black } \\
\text { Content (phr) }\end{array}$ & $\begin{array}{c}\sigma \\
(\mathrm{MPa})\end{array}$ & $\begin{array}{c}\tau \\
(\mathrm{min} .)\end{array}$ & $\beta(\mathrm{t})$ & $\Delta$ & $\begin{array}{c}\eta_{3} \\
(\mathrm{GPa} . \mathrm{sec})\end{array}$ \\
\hline \multirow{3}{*}{30} & 0.98 & 17.45 & $09.19 \times 10^{5}$ & 0.20 & 17.21 \\
\cline { 2 - 6 } & 1.96 & 32.89 & $23.39 \times 10^{5}$ & 0.15 & 13.07 \\
\cline { 2 - 6 } & 2.94 & - & - & - & - \\
\hline \multirow{3}{*}{40} & 1.96 & 158.70 & $06.78 \times 10^{5}$ & 0.28 & 25.66 \\
\cline { 2 - 6 } & 2.94 & 170.30 & $18.50 \times 10^{5}$ & 0.20 & 21.72 \\
\cline { 2 - 6 } & 3.92 & - & - & - & - \\
\hline \multirow{3}{*}{50} & 0.98 & 84.03 & $06.05 \times 10^{5}$ & 0.23 & 9.41 \\
\cline { 2 - 6 } & 1.96 & 113.63 & $07.15 \times 10^{5}$ & 0.29 & 6.41 \\
\cline { 2 - 6 } & 2.94 & 156.25 & $13.05 \times 10^{5}$ & 0.16 & 8.82 \\
\hline
\end{tabular}

The retardation time $\tau$ increases to maximum at carbon black content 40 phr and then decreases with increasing HAF content under all different applied stresses. This is attributed to the formation of the compact structure at $40 \mathrm{phr}$ carbon black which is considered to be responsible for the hindering of the sliding of macromolecules over each other.

Another parameter which gives an indication of the previous history of the deformation gradients is the memory function $\beta(\mathrm{t})$. It is manifested during the time dependent recoverable strain and is given by $\beta(\mathrm{t})=\varepsilon_{\mathrm{st}} / \varepsilon_{\mathrm{elr}}$ [23]. Since the creep rate depends on the value of the applied stress and carbon black concentration; the memory function should also depend on them, besides the time of the creep process. From Table (3), it is noticed that $\beta(t)$ decreases with increasing HAF content at certain applied stress while it increases with increasing the applied stress at certain HAF content. This might be due to the increase of cross-linking density. It is also clear that the relaxation strength $\Delta$ vary with the HAF black content and the applied stress. It has been noticed that, $\Delta$, increases with increasing carbon black content under certain applied stress and decreases with increasing the applied stress for certain HAF content up to $40 \mathrm{phr}$ carbon content which is not the case above $40 \mathrm{phr}$ carbon content.

After long time $\left(t_{1}\right)$ from applying the stress $\sigma$, the dashpot $\eta_{3}$ becomes 
responsible for the creep rate and recoverable elastic strain, so the viscosity at plastic deformation $\eta_{3}$ can be calculated from the relation $\eta_{3}=\left(\sigma / \varepsilon_{\text {irr }}\right) t_{1}$ [13]. From Table (3), it has been noticed that, $\eta_{3}$ increases with increasing HAF black content and reaches maximum value for the sample containing $40 \mathrm{phr}$ of HAF black as a result of the formation of a compact structure.

\section{Conclusion:}

Variations in the mechanical properties of 50NBR/50EPDM blend obtained from stress- strain and creep measurements show an enhancement on the addition of HAF carbon black. The incorporation of HAF/black increases the stiffness, young's modulus, rupture stress, elastic moduli, viscosity, retardation time and the relaxation strength of the blend. Each of these parameters shows a maximum around $40 \mathrm{phr}$ of HAF/black. However, both of the rupture strain and the activation volume show the opposite behaviour. A compact structure was believed to be around the concentration of $40 \mathrm{phr} \mathrm{HAF}$ black which is responsible of the maxima and minima in all the above parameters. The analysis of the stress - strain behaviour of the tested samples revealed that the visco-elastic behaviour follows the standard linear solid model. The calculated parameters by this model were fairly consistent with the experimental data.

\section{Acknoledgement}

The author thanks Prof. Dr F. Abd-El Salam for his valuable help and support during the period of this work.

\section{References}

1. J. P. Donnet and E. Custodero, Carbon 30, 813 (1992).

2. M. J. Wang, S. Wolff and J.P. Donnet, Rub. Chem. Tech. 64, 559 (1991).

3. Y.S. Zuyev, Polym. Sci., (USSR), 221, p. 1315 (1980).

4. B. Banerjee and W.Millns; $5^{\text {th }}$ Conf. Eur. Plast. Caoutch, 2, D7-1-D7-6 (Eng), (1978).

5. F. Abd-El Salam, M. H. Abd-El Salam, M. T. Mostafa, M. R. Nagy, M. I. Mohamed J. Appl. Polym. Sci. 90, 1539, (2003).

6. M. Abu -Abdeen; J. Appl. Polym. sci . 81 (9), 2265 (2001).

7. M. A. Fahim, M. Roshdy and H.M. Osman; Proc. Math. Phys. Soc. Egypt. 64, 37 (1987).

8. A. El-Lawindy and S. El-Guiziri, J. Phys. D: App. Phys., 33, 1894 (2000).

9. A. El-Lawindy, Polymer Composites, 24, 94 (2003). 
10. M. H. Abd El-Salam, Ph.D. Thesis, Fac- Sci., Cairo univ. Cairo, Egypt, p.41 (1997).

11. S. Govindjee and J. Simo, J. Mech. Phys. Sol. 39, 87 (1991).

12. A. Tager, "Physical Chemistry of polymers", Mir Pub., 2nd Ed., Moscow, (1978).

13. L.E. Nielsen and R.F. Landel, "Mechanical Properties of Polymers and Composites", 2nd ed., Revised and Expanded, (1994).

14. R.G.C. Arridge, "Mechanics of Polymers", Oxford Univ. Press, (1975).

15. H. S. Kaufman, "Introduction to Polymer and Technology", John Wiley \& Sons: New York; (1981).

16. C.E. Locke and D.R. Paul; Polym. Eng. Sci. 13, p.308 (1973).

17. L. H. Sperling, "Introduction to physical polymer science", New York: Wiley; (1986).

18. N. E. Marcovich, M. A.Villar, J Appl Polym Sci. 90 (10), 2775 (2003).

19. [19] V.P. Cyras, J.F. Martucci, S. Iaanace, A. Vazquez, J. Thermoplastic Comp. Mat. 15, 253 (2002)

20. S. Hamza and H.M. Osman; Polymer Bulletin 12, p. 209

21. G.V.Vinogradov and A.YA.Malkin, "Rheology of Polymers", Mir Pub., Moscow (1980).

22. W.R. Cannon and O.D. Sherby, Metallurg .Trans. 1, 1031 (1970).

23. H. Henning Kausch, J.A. Hassel and R.I. Jaffee, "Deformation and Fracture of high Polymers", Plenum Press, (1973). 